[6] N. Dimov et I. Salicev. La valorisation du lait de brebis. Zemizdat. Edition d'Etat pour la littérature agricole. 1957. Traduit du bulgare, 116 p., 90.

[7] R. Marracino. Metodi di lavorazione del latte di pecora, di capra e di bufala in Italia. Il Latte, 31. Septembre 1957, n० 9. 603-610.

[8] G. Roeder. Grundzüge der Milchwirtschaft und des Molkereiwesens. 1954. Ed. P. Parey. 830 p., 679.

\title{
LA RECHERCHE DU LAIT DE VACHE AJOUTÉ AU LAIT DE CHËVRE. APPLICATION AU CAS DU FROMAGE (1)
}

par

\author{
S. KUZDZAL-SAVOIE et W. KUZDZAL \\ Station Centrale de Recherches laitières, \\ et de Technologie des Produits animaux. Jouy-en-Josas
}

\section{I. - INTRODUCTION}

Une proportion d'acides gras volatils élevée et nettement supérieure à celle de la matière grasse du lait de vache caractérise la matière grasse du lait de chèvre. Utilisant cette propriété, CHollet et CAmus (1) d'une part et Lopez [2] d'autre part, ont proposé deux méthodes permettant de déceler la présence de lait de vache dans le lait de chèvre. La méthode de Chollet et CAmus [1] a été également appliquée à l'étude des fromages de chèvre.

Une méthode sérologique a été proposée, en 1952, par HADLAND et Solberg [3]. Elle permet de déceler la présence de 1\% de lait de vache dans le lait de chèvre, qu'il s'agisse de lait entier ou de lait écrémé. Cependant cette méthode ne paraît pas jusqu'à maintenant être applicable au fromage.

La méthode que nous proposons ci-dessous (applicable au lait et au fromage) repose sur l'observation suivante : le lait de chèvre ne contient pas de $\beta$ carotène [4], alors que ce pigment liposoluble est au contraire toujours présent dans le lait de vache. Le colostrum de chèvre peut contenir du $\beta$ carotène [4] mais ceci ne représente pas un inconvénient sérieux, le colostrum ne devant pas être incorporé au lait commercialisé.

La méthode est applicable au fromage car il a été établi [5] que la concentration en carotène $d u$ fromage ne varie pas sensiblement au cours de l'affinage.

(1) Ann. Tech. I. N. R. A., 1959, 2, 131. 
Ainsi il suffit de s'assurer de l'absence de $\beta$ carotène dans le lait ou le fromage de chèvre pour affirmer qu'il s'agit de produits " pur chèvre").

\section{II. - JUSTIFIGATION DE LA MÉTHODE}

Le $\beta$ carotène en solution dans l'hexane possède un spectre d'absorption caractérisé par trois maxima correspondant aux longueurs d'onde suivantes : $425 \mathrm{~m} \mu, 450 \mathrm{~m} \mu, 477 \mathrm{~m} \mu$. Le pic que présente la courbe d'absorption à $450 \mathrm{~m} \mu$ est particulièrement net.

La coloration de la matière grasse du lait de vache est due principalement au $\beta$ carotène. Si l'on mesure l'absorption en lumière visible de la matière grasse du lait de vache en solution dans un solvant organique ( $5 \mathrm{~g}$ de matière grasse dans $10 \mathrm{ml}$ d'hexane), on retrouve le spectre caractéristique du $\beta$ carotène. Si l'on effectue la même mesure sur la matière grasse du lait de chèvre on ne voit apparaître aucun pic, (absence de $\beta$ carotène).

La figure 1 montre l'aspect des courbes obtenues.

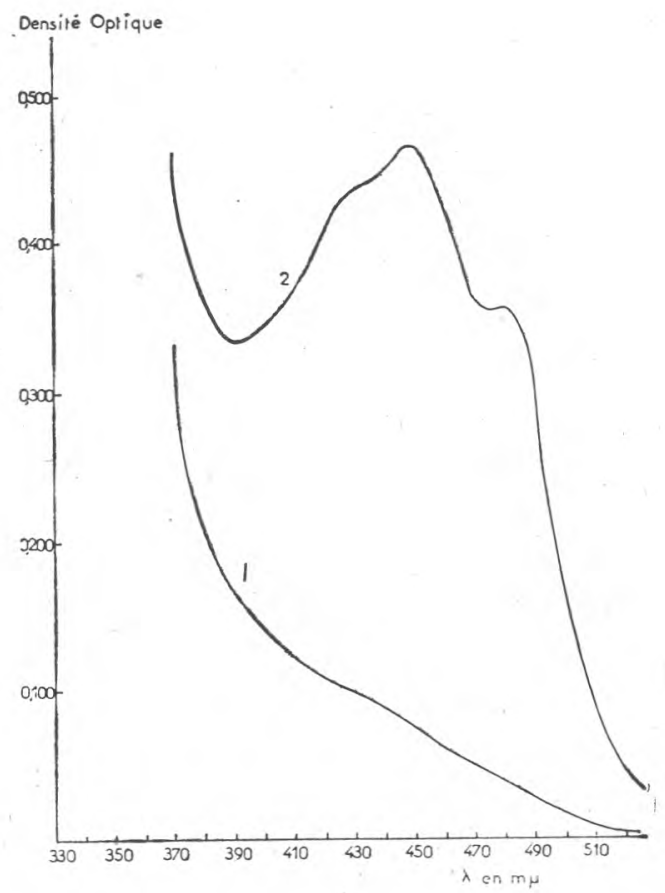

Fig. 1. - Spectres d'absorption de solutions de matiòre grasse dans l'hexane. 1 : lait de chèvre ; 2 : lait de vache. 
On peut ainsi mettre en évidence, très rapidement, la présence de lait de vache dans le lait de chèvre par la simple observation du spectre de la matière grasse : il suffit en effet de noter l'amorce du spectre caractéristique du $\beta$ carotène pour affirmer la présence de lait de vache.

Cette méthode présente cependant deux inconvénients : tout d'abord elle nécessite l'utilisation d'un spectrophotomètre et ensuite, le fond spectral obtenu est très important.

Pour pallier le premier inconvénient, on a pensé qu'un dosage colorimétrique de l'insaponifiable de la matière grasse de lait de vache ou de lait de chèvre pouvait conduire à des résultats intéressants. En fait l'insaponifiable de la matière grasse de lait de chèvre est toujours coloré, quoique faiblement par rapport à l'insaponifiable de la matière grasse de lait de vache. En se basant, pour évaluer le contenu en carotène sur une courbe d'étalonnage établie à partir d'une solution chloroformique de $\beta$ carotène pur (HoFrmanNLAROCHE) à des concentrations variables (lectures au colorimètre tricellule JoBıN et YvoN), on a trouvé en août 1955 sur des laits de mélange provenant d'une part du troupeau de chèvres et d'autre part du troupeau de vaches élevés au Centre National de Recherches Zootechniques, une valeur correspondant à $30-50 \mu \mathrm{g}$ de $\beta$ carotène pour $100 \mathrm{~g}$ de matière grasse du lait de chèvre contre 620 à $650 \mu \mathrm{g}$ pour $100 \mathrm{~g}$ de matière grasse de lait de vache. Une extraction supplémentaire de l'insaponifiable par l'alcool méthylique à $90 \%(v / \nabla)[6]$ destinée à séparer les caroténols (xanthophylles en particulier) n’á pas permis d'éliminer cette légère coloration. Nous avons done finalement adopté une méthode de séparation chromatographique de l'insaponifiable sur colonne d'alumine, avee élutions successives par des solvants différents [7]. Cette méthode permet d'isoler le $\beta$ carotène des autres constituants colorés de l'insaponifiable; le $\beta$ carotène est en effet le premier composé coloré élué. On peut ensuite, soit étudier le spectre d'absorption (de 350 à $500 \mathrm{~m} \mu$ ) de la solution obtenue, soit plus simplement mesurer son absorption avee un écran bleu au photocolorimètre.

Dans l'application que nous avons faite de cette méthode, nous avons utilisé la spectrophotométrie. On obtient des spectres d'absorption bien caractérisés, avec un fond spectral réduit au minimum.

\section{III. - TEGHNIQUE EXPÉRIMENTALE}

Le $\beta$ carotène est isolé de l'insaponifiable de la matière grasse du lait, du beurre ou du fromage, par chromatographie d'adsorption sur colonne d'alumine. Il est ensuite caractérisé par son spectre d'absorption en lumière visible. 
Il est donc nécessaire de réaliser les opérations suivantes : saponification de la matière grasse, extraction de l'insaponifiable, chromatographie sur alumine, mesure de l'absorption de la solution de $\beta$ caortène par spectrophotomètrie.

\section{A. - Saponification}

La saponification peut être effectuée soit directement, soit indirectement, après extraction de la matière grasse.

1. - Cas du lait :

a) Saponification directe [8] : mesurer $100 \mathrm{ml}$ de lait, les verser dans un erlenmeyer de $500 \mathrm{ml}$. D'autre part dissoudre $20 \mathrm{~g}$ de potasse caustique en pastilles dans un mélange de $10 \mathrm{ml}$ d'eau distillée et $100 \mathrm{ml}$ d'alcool éthylique. Verser cette solution dans l'erlenmeyer contenant le lait. Agiter légèrement et porter à ébullition modérée au bain-marie sous réfrigérant à reflux. Maintenir l'ébullition pendant deux heures. Laisser refroidir.

b) Saponification indirecte : après écrémage du lait et fabrication du beurre (voir cas du beurre).

2. - Cas du beurre : peser $5 \mathrm{~g}$ de matière grasse obtenue après fusion et centrifugation du beurre. Ajouter $25 \mathrm{ml}$ d'une solution alcoolique de potasse environ $2 \mathrm{~N}$ et saponifier comme précédemment.

3. - Cas du fromage :

a) Saponification directe : peser un poids de fromage correspondant à environ $5 \mathrm{~g}$ de matière grasse dans un erlenmeyer de $500 \mathrm{ml}$. Ajouter $30 \mathrm{~g}$ de potasse caustique en pastilles, préalablement dissous dans $15 \mathrm{ml}$ d'eau distillée et $150 \mathrm{ml}$ d'alcool éthylique. Saponifier au bain-marie comme précédemment.

b) Saponification indirecte : on extrait la matière grasse du fromage par la méthode de SoxHLET à l'éther de pétrole après avoir intimement mélangé le fromage à son poids de sulfate de soude anhydre et à son poids de sable de Fontainebleau. Après extraction pendant 24 heures, on filtre l'extrait éthéré. Le solvant est évaporé si nécessaire et on amène la solution à un volume de $100 \mathrm{ml}$, avec l'éther de pétrole. On prélève trois fois $5 \mathrm{ml}$ qu'on laisse écouler dans trois capsules tarées. Ces capsules sont placées dans une étuve Chopin. Le solvant est évaporé à $60^{\circ} \mathrm{C}$ sous pression réduite (quelques $\mathrm{cm}$ de mercure). Le poids d'extrait sec permet de connaître le poids de matière grasse contenue dans le poids de fromage utilisé pour l'extraction. On prélève en même temps $75 \mathrm{ml}$ que l'on place dans un erlenmeyer de $300 \mathrm{ml}$. Après l'évaporation du solvant on ajoute $25 \mathrm{ml}$ de potasse alcoolique $2 \mathrm{~N}$ environ et l'on porte au 
bain-marie; on amène à ébullition et on maintient l'ébullition pendant deux heures.

\section{B. - Extraction de l'insaponifiable}

On peut extraire l'insaponifiable soit directement sur le produit résultant de la saponification, (cas du lait et du fromage ayant subi l'attaque alcaline), soit après avoir amené la solution de savon aux proportions fixées par LEMELAND [9], e'est-à-dire alcool à 50\% et potasse $0,1 \mathrm{~N}$. Ceci est aisé à réaliser lorsqu'on a utilisé la solution de potasse environ $2 \mathrm{~N}$ pour la saponification. Il suffit en effet de neutraliser l'excès de potasse, avec l'acide chlorhydrique environ $\mathrm{N}$, d'ajouter ensuite une quantité de potasse permettant d'obtenir le titre $0,1 \mathrm{~N}$ et d'amener la solution au volume final choisi (avec de l'eau et éventuellement de l'alcool). La solution de savon est alors épuisée par trois fois $50 \mathrm{ml}$ d'éther de pétrole. Les extraits éthérés sont réunis et lavés à l'eau avec faible agitation. On ajoute ensuite environ $10 \mathrm{~g}$ de sulfate de soude anhydre à la solution d'insaponifiable et après une heure de contact la solution est filtrée et le solvant évaporé, jusqu'à volume réduit (quelques $\mathrm{ml}$ ). L'insaponifiable est repris par l'éther de pétrole et transvasé en capsule tarée. L'évaporation est terminée à la température ambiante au dessicateur sous vide. L'insaponifiable est aussitôt pesé et repris par quelques $\mathrm{ml}$ d'hexane (le moins possible).

\section{C. - Chromatographie}

La méthode de séparation chromatographique du $\beta$ carotène est celle décrite par Thompson et Kon [7]. On utilise de l'alumine standardisée selon BRockmann. Cette alumine est activée par passage de quelques heures au four (par exemple un passage de 6 heures à $600^{\circ} \mathrm{C}$ ) suivi d'une réhumidification à $4 \%(\mathrm{p} / \mathrm{p})$.

La colonne est un tube de verre de $13 \mathrm{~cm}$ de long et de $1,3 \mathrm{~cm}$ de diamètre intérieur. Ce tube est prolongé par un petit tube de $0,4 \mathrm{~cm}$ de diamètre intérieur et de 3 ou $4 \mathrm{~cm}$ de long. Cette colonne est montée sur une fiole à vide. On place à la base de la colonne un tampon de coton hydrophile de $1 \mathrm{~cm}$ de haut et l'on verse l'alumine en suspension dans l'hexane. On laisse écouler librement. La hauteur de la colonne d'alumine doit atteindre $4 \mathrm{~cm}$. une rondelle de papier filtre est placée sur la colonne. La surface de la colonne doit toujours être couverte de solvant.

On verse à la pipette la solution d'insaponifiable. On la laisse pénétrer dans la colonne. On rince ensuite les bords de la colonne par de l'hexane et l'on fait passer encore quelques $\mathrm{ml}$ d'hexane. 
On élue ensuite dans une fiole propre, le $\beta$ carotène entraîné sélectivement sous forme d'un anneau orange, par une solution d'hexane contenant $0,75 \%(v / v)$ d'acétone. On arrête l'élution après passage de l'anneau orange. Lorsque l'analyse est effectuée sur du lait ou du fromage de chèvre, on ne voit pas cet anneau orange.

D'autres anneaux colorés restent sur le sommet de la colonne et ne sont élués qu'arec de l'hexane contenant $3 \%(\nabla / \nabla)$ d'acétone ou $8 \%$ d'éthanol $(\nabla / v)$.

Ces autres anneaux colorés sont identiques pour les deux espèces chèvre et vache. Ils sont jaunes dans le cas du lait jaune, rose et brun dans celui du fromage.

Après l'évaporation du solvant on reprend le résidu par l'hexane pur et on amène à un volume de 10 ou $20 \mathrm{ml}$ suivant le concentration.

\section{D. - Mesure spectrophotométrique}

L'absorption de la solution est mesurée de $350 \mathrm{~m} \mu$ à $500 \mathrm{~m} \mu$ (spectrophotomètre Beckman D. U., cuve de $1 \mathrm{~cm}$ ).

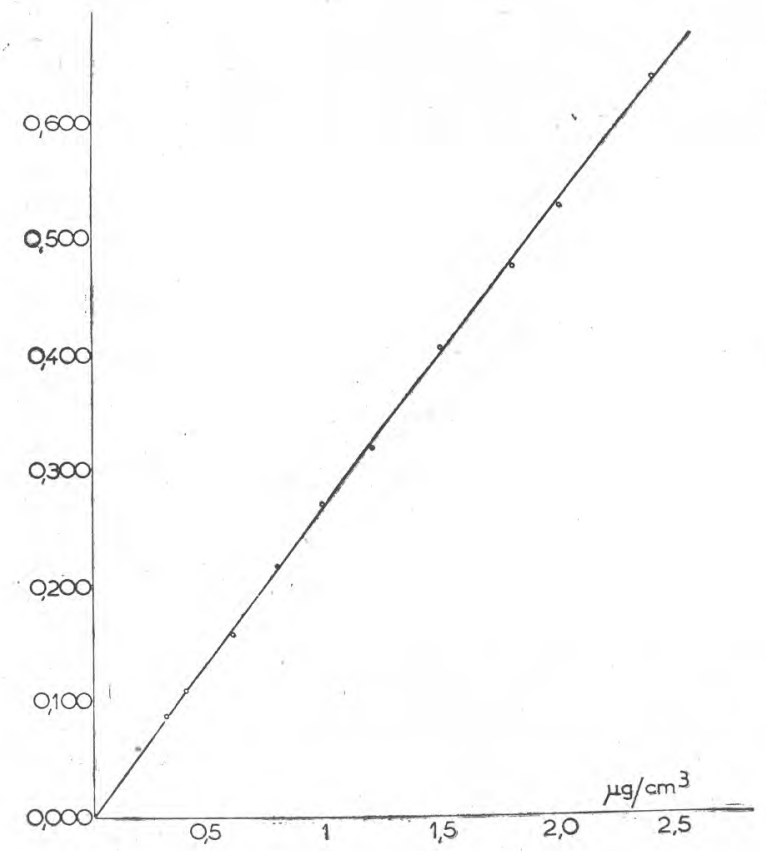

Fig. 2. - Courbe d'étalonnage : densités optiques mesurées à $4.500 \mathrm{~A}^{\circ}$. Solutions à concentrations variables de $\beta$ carotène (HoffMann-Laroche). 
La forme de la courbe d'absorption obtenue permet de reconnaître la présence ou l'absence de $\beta$ carotène.

Pour déterminer la concentration en $\beta$ carotène on observe la valeur de la densité optique au maximum de la courbe, et l'on se reporte à une courbe d'étalonnage établie en mesurant à $450 \mathrm{~m} \mu$ l'absorption de solutions de $\beta$ carotène pur (HoFFMANN-LaRoche) de concentrations variables, de zéro à $2 \mu \mathrm{g}$ par mlde solution. Pour ces faibles concentrations, l'absorption est proportionnelle à la concentration et la courbe d'étalonnage est une droite (fig. 2).

\section{E. - Mesure colorimétrique}

Elle peut être utilisée si l'on ne dispose pas de spectrophotomètre. On examine la solution d'hexane contenant ou non du $\beta$ carotène sous écran bleu. La concentration en $\beta$ carotène est déterminée par référence à une courbe d'étalonnage.

\section{F. - Expression des résultats}

Les résultats sont exprimés en $\mathrm{mg}$ ou $\mu \mathrm{g}$ de $\beta$ carotène pour $100 \mathrm{~g}$ de matière grasse. Dans les cas où l'on utilise la saponification directe il est done nécessaire de déterminer sur un autre échantillon le taux de matière grasse du produit étudié (lait ou fromage).

Remarque : Le carotène s'oxyde facilement. Sa coloration disparaît alors. Le contact de l'air, l'exposition à la lu mière, les températures élevéels, l'acidité du milieu favorisent cette oxydation. Les opérations du dosage ci-dessus décrit doivent donc être menées le plus possible à l'abri de la lumière; le temps pendant lequel le carotène se trouve non dissous doit être réduit au maximum; l'eau du bain-marie utilisée pour la saponification est portée à une température voisine de $85^{\circ} \mathrm{C}$.

\section{IV. - APPLIGATION DE LA MÉTHODE}

\section{A. - Dosage de $\beta$ carotène dans le lait}

Les échantillons sont constitués par des laits de mélange provenant d'une part du troupeau de chèvres et d'autre part du troupeau de vaches élevés au Centre National de Recherches Zootechniques. Le $\beta$ carotène est dosé après saponification directe de la matière grasse du lait.

Le 20 janvier 1958 le lait de vache contenait $180 \mu \mathrm{g}$ de $\beta$ carotène par litre de lait ou $493 \mu \mathrm{g}$ de $\beta$ carotène pour $100 \mathrm{~g}$ de matière grasse.

Les spectres d'absorption obtenus sont représentés sur la figure 3 . 


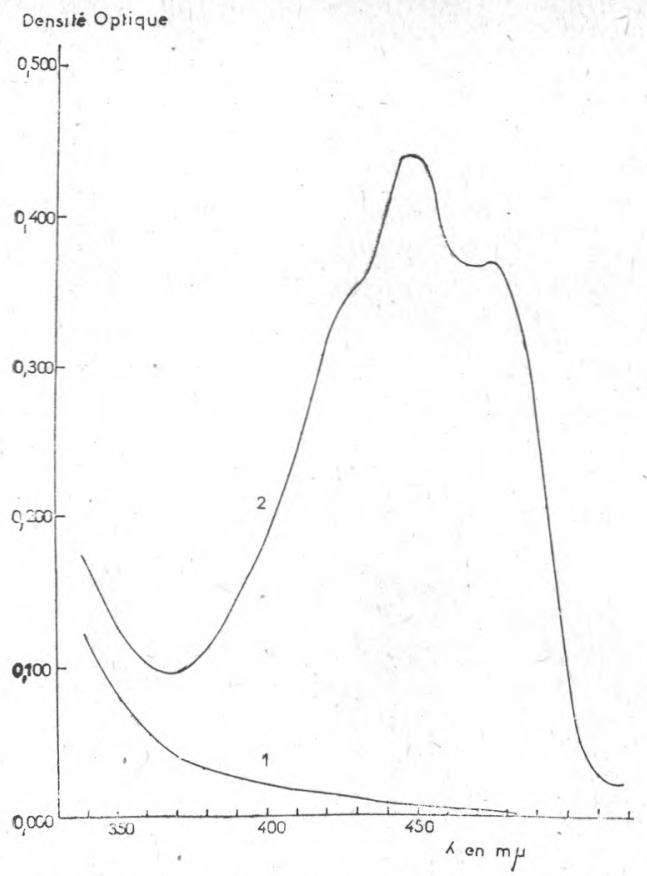

Fig. 3. - Spectres d'absorption d'éluats d'insaponifiables de la matière grasse de lait de chèvre (1) et de lait de vache (2).



Fig. 4. - Spectres d'absorption d'éluats d'insaponifiables de la matière grasse du beurre. 1 : beurre de chèvre pur (échantillon I). -2 et 3 : beurre de chèvre et de vache mélangés (échantillons II et III). - 4 : beurre de vache pur (échantillon IV). 


\section{B. - Dosage de $\beta$ carotène dans le beurre}

On prépare du beurre de vache et du beurre de chèvre. Après fusion et centrifugation séparées de ces deux beurres, on pèse quatre échantillons de matière grasse (les échantillons I, II, III et IV) sur lesquels on dose le $\beta$ carotène. La nature des échantillons et les résultats de l'analyse sont indiqués dans le tableau I. Les spectres d'absorption sont représentés sur la figure 4.

On retrouve dans les échantillons II et III, avec une erreur relative de l'ordre de $1 \%$, les quantités de $\beta$ carotène calculées. On utilise pour ce calcul la quantité de $\beta$ carotène dosée dans l'échantillon IV : beurre de vache.

\section{TABLEAU I}

DOSAGE DE $\beta$ GAROTENE DANS LE BEURRE

\begin{tabular}{|c|c|c|c|c|c|}
\hline \multirow[t]{2}{*}{, } & \multicolumn{2}{|c|}{$\begin{array}{l}\text { Poids (en g) } \\
\text { de matière grasse } \\
\text { de lait }\end{array}$} & \multicolumn{2}{|c|}{$\begin{array}{c}\text { Quantité de } \beta \text { carotène } \\
\text { exprimée en } \mu \mathrm{g} \\
\text { p. } 100 \mathrm{~g} \text { de matière grasse }\end{array}$} & \multirow{2}{*}{$\begin{array}{c}\text { Erreur } \\
\text { relative } \\
\%\end{array}$} \\
\hline & de chèvre & de vache & dosée & calculée & \\
\hline Echantillon I .. & 5 & 0 & 0 & & 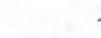 \\
\hline Echantillon II. & 3,750 & 1,280 & 119,3 & 120,3 & 0,9 \\
\hline Echantillon III. & 2,501 & 2,556 & 237,3 & 240,3 & 1,3 \\
\hline Echantillon IV. & 0 & 5 & 470,0 & & \\
\hline
\end{tabular}

\section{C. - Dosage de $\beta$ carotène dans le fromage}

On détermine le taux butyreux du lait de vache et du lait de chèvre purs dont on dispose. On incorpore ensuite à deux échantillons de lait de chèvre, des quantités de lait de vache variables avec le taux butyreux des deux laits, mais calculées de telle façon que l'on obtienne, d'une part un lait dont la matière grasse est constituée par 25 parties de matière grasse de vache et 75 parties de matière grasse de chèvre (mélange II), et, d'autre part, un lait dont la matière grasse est constituée en parties égales de matière grasse de chèvre et de matière grasse de vache (mélange III).

A partir de ces quatre échantillons de lait (lait de chèvre pur (I), lait de vache pur (IV), mélanges II et III) on fabrique des fromages suivant la technique habituelle de fabrication des fromages de. chèvre à la ferme

L'e tableau II rend compte des résultats obtenus au cours de trois expériences (A, B, C). Dans les expériences A et B la matière 
TABLEAU II



$1^{\text {re }}$ partie : modalités de fabrication des fromages

\begin{tabular}{|c|c|c|c|c|c|c|c|c|}
\hline & \multirow{2}{*}{$\begin{array}{c}\text { Date } \\
\text { de fabrication } \\
\text { des fromages }\end{array}$} & \multicolumn{2}{|c|}{ Taux butyreux du lait } & \multicolumn{5}{|c|}{ Quantité de lait (en litres) mis en œuvre dans la fabrication des fromages } \\
\hline & & Chèvre & Vache & & $\begin{array}{c}\mathrm{I} \\
\text { (pur) }\end{array}$ & $\begin{array}{c}\text { II } \\
\text { (mélangé) }\end{array}$ & $\begin{array}{c}\text { III } \\
\text { (mélangé) }\end{array}$ & $\begin{array}{l}\text { IV } \\
\text { (pur) }\end{array}$ \\
\hline Expérience A .... & $21-10-57$ & 34 & 40 & $\begin{array}{l}\text { lait de chèvre } \\
\text { lait de vache }\end{array}$ & $\begin{array}{l}2 \\
0\end{array}$ & $\begin{array}{l}1,5 \\
0,425\end{array}$ & $\begin{array}{l}1 \\
0,85\end{array}$ & $\begin{array}{l}0 \\
2\end{array}$ \\
\hline Expérience B .... & $9-12-57$ & 42,5 & 32,5 & $\begin{array}{l}\text { lait de chèvre } \\
\text { lait de vache }\end{array}$ & $\begin{array}{l}1 \\
0\end{array}$ & $\begin{array}{l}0,75 \\
0,327\end{array}$ & $\begin{array}{l}05 \\
0,653\end{array}$ & $\begin{array}{l}0 \\
1\end{array}$ \\
\hline Expérience C .... & $21-10-57$ & 34 & 40 & $\begin{array}{l}\text { lait de chèvre } \\
\text { lait de vache }\end{array}$ & $\begin{array}{l}2 \\
0\end{array}$ & $\begin{array}{l}1,5 \\
0,425\end{array}$ & $\begin{array}{l}1 \\
0,85\end{array}$ & $\begin{array}{l}0 \\
2\end{array}$ \\
\hline
\end{tabular}

2 e partie : résultats de l'analyse

\begin{tabular}{|c|c|c|c|c|c|c|c|c|c|}
\hline \multirow[t]{3}{*}{1} & \multirow{3}{*}{$\begin{array}{c}\text { Date } \\
\text { de l'analyse } \\
\text { des fromages } \\
\text { affinés }\end{array}$} & \multicolumn{6}{|c|}{ Quantité de $\beta$ carotène exprimée en $\mu \mathrm{g}$ pour $100 \mathrm{~g}$. de matière grasse } & \multirow{2}{*}{\multicolumn{2}{|c|}{ Erreur relative \% }} \\
\hline & & \multicolumn{4}{|c|}{ dosée } & \multicolumn{2}{|c|}{ calculée } & & \\
\hline & & $\begin{array}{c}\text { I } \\
\text { (chèvre) }\end{array}$ & $\begin{array}{c}\text { II } \\
\text { (mélangé) }\end{array}$ & $\begin{array}{c}\text { III } \\
\text { (mélangé) }\end{array}$ & $\begin{array}{c}\text { IV } \\
\text { (vache) }\end{array}$ & II (1) & III (1) & II & III \\
\hline Expérience A. & $15-11-57$ & 0 & 118,5 & 247,2 & 509,6 & 127,4 & 254,8 & $-7,0$ & $-3,0$ \\
\hline Expérierce B. & $17-12-57$ & 0 & 116,0 & 220,6 & 493,0 & 123,2 & 246,5 & $-5,7$ & $-10,5$ \\
\hline Expérience C .. & $28-1-58$ & 0 & 73,5 & 168,4 & 327,6 & 81,9 & 163,8 & $-10,2$ & $+\quad 2,7$ \\
\hline
\end{tabular}

(1) La matière grasse du lait, ayant servi à la fabrication des fromages II et III contient, respectivement, 25 et $50 \%$ de matière grasse de lait de vache : par conséquent le taux de $\beta$ carotène de la matière grasse des fromages II et III devrait correspondre à 25 et $50 \%$ du taux de $\beta$ carotène de la matière grasse du fromage fabriqué à partir de lait de vache pur. 




Fig. 5. - Expérience A : Spectres d'absorption d'éluats d'insaponifiables de la matière grasse de fromages fabriqués. 1 : avec du lait de chèvre pur (I) ; 2 : avec du lait de mélange (II) ; 3 : avec du lait de mélange (III) ; 4 : avec du lait de vache pur (IV). - Nota : éluats 1 et 2 repris par $10 \mathrm{ml}$ d'hexane; éluats 3 et 4 repris par $20 \mathrm{ml}$ d'hexane.

grasse sur laquelle est effectué le dosage de $\beta$ carotène est extraite des fromages (20 g) par la méthode de Soxhlet (à l'éther de pétrole). Dans l'expérience $C$, le taux de matière grasse est déterminé par une méthode butyrométrique (VAN GULIK) et le dosage du $\beta$ carotène est effectué en utilisant la méthode de saponification alcaline directe (sur $10 \mathrm{~g}$ de fromage).

Les spectres d'absorption étudiés sont portés sur les figures 5 (expérience A), 6 (expérience B) et 7 (expérience C). Le dosage du $\beta$ carotène est effectué sur un poids variable de matière grasse. C'est pourquoi sur les figures 5, 6 et 7, les rapports des ordonnées des courbes 2 et 3 à l'ordonnée de la courbe 4 (évaluées au maximum d'absorption) ne sont qu'approximativement égaux à 25 et $50 \%$. 


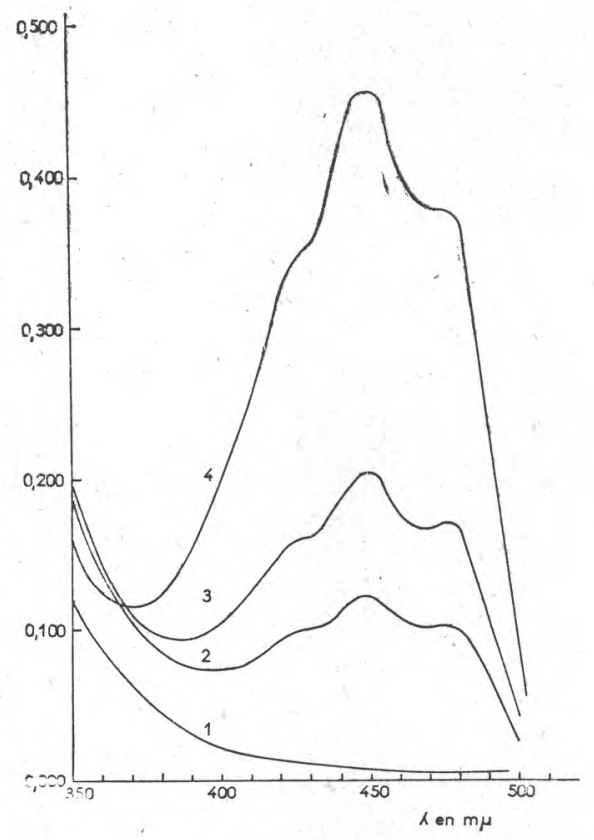

Fig. 6. - Expérience B : Spectres d'absorption d'éluats d'insaponifiables (repris par $10 \mathrm{ml}$ d'hexane) de matière grasse de fro mages fabriqués dans les conditions indiquées dans la légende de la figure 5.



Fig. 7. - Expérience C: Spectres d'absorption d'éluats d'insaponifiables (repris par $10 \mathrm{ml}$ d'hexane) de la matière grasse de fromages fabriqués dans les conditions indiquées dans la légende de la figure 5. L'insaponifiable est extrait après attaque alcaline. 
Le tableau II montre que l'on retrouve une quantité de $\beta$ carotène généralement inférieure à celle prévue par le calcul; on utilise pour ce calcul, la quantité de $\beta$ carotène dosée dans la matière grasse du fromage fabriqué à partir de lait de vache pur. La différence peut atteindre $10 \%$.

La fidélité de la méthode est donc moins bonne dans le cas des fromages que dans le cas du beurre. En fait, les causes d'erreur dans le cas des fromages sont aussi plus nombreuses. Alors que pour le beurre on constitue les mélanges de matière grasse de chèvre et de vache par pesée d'une quantité convenable de chacune de ces matières grasses, ces proportions sont établies, pour les fromages, par un mélange de lait de chacune des espèces. Il n'est pas impossible que suivant les conditions du moulage, de l'égouttage, etc. une modification des proportions relatives des deux matières grasses intervienne dans le fromage final.

De plus la quantité de matière grasse extraite par la méthode de SoxHLET est généralement inférieure à celle que l'on obtiendrait en pratiquant sur le même échantillon l'extraction de la matière grasse après attaque acide (procédé S.B.R. par exemple) du fromage; mais on ne peut employer cette dernière méthode car nous avons vérifié que l'attaque acide détruit complètement le $\beta$ carotène.

Dans les analyses qui ont été rapportées ci-dessus, le maximum d'absorption du composé élué par la solution d'hexane contenant $0,75 \%(\mathrm{v} / \mathrm{v})$ d'acétone se place souvent vers $448 \mathrm{~m} \mu$. Cetécart avec la valeur trouvée pour le $\beta$ carotène pur $(450 \mathrm{~m} \mu)$ peut provenir d'une part d'une petite quantité $d$ ' $\alpha$ carotène élué en même temps que le $\beta$ carotène et dont le maximum d'absorption se place à $445 \mathrm{~m} \mu$ en solution dans l'hexane (10a) et d'autre part de l'existence de dérivés d'oxydation du $\beta$ carotène formés au cours des opérations préliminaires d'extraction de l'insaponifiable ; ces composés, en solution dans l'hexane présentent un maximum d'absorption à une longueur d'onde légèrement inférieure $(446 \mathrm{~m} \mu)(10 \mathrm{~b})$.

\section{V. - DISGUSSION ET GONGLUSION}

Ni la méthode que nous proposons, ni d'ailleurs la méthode de Chollet et Camus (1) ne sont applicables à la recherche d'une fraude qui s'exercerait sur le lait écrémé. D'autre part, comme on l'observe pour tous les constituants du lait et en particulier pour l'acidité volatile soluble et insoluble de la matière grasse du lait [11], [12], [13] la teneur moyenne du lait de vache en $\beta$ carotène peut varier suivant la race des vaches laitières, le stade de lactation, la nature des pâturages, le mode de vie et d'alimentation du cheptel bovin. Les variations saisonnières du contenu en carotène du lait de 
vache ont été étudiées dans différents pays [14], [15], [16]. En France, cette étude a été limitée à la région parisienne [8]. Mais il est toujours possible de connaître, à une époque déterminée et dans une région déterminée, le contenu en $\beta$ carotène d'un lait de vache de mélange utilisé comme lait de référence. Il est également facile d'analyser en même temps qu'un fromage de chèvre, un fromage fabriqué avec du lait de vache dans la même région et à la même époque que le fromage de chèvre suspecté.

L'intérêt essentiel de la méthode proposée et l'avantage qu'elle présente par rapport à la méthode de CHOLLet et CAMUS [1] réside dans le fait que la comparaison porte, non pas, comme dans le cas des acides volatils sur des composés dont les proportions relatives seules sont différentes dans le lait de vache et dans le lait de chèvre, mais sur un corps, le $\beta$ carotène, présent dans le lait de vache et complètement absent dans le lait de chèvre.

\section{RÉFÉRENCES BIBLIOGRAPHIQUES}

[1] A. Chollet et A. CAmus. Etude de la matière grasse du lait de chèvre, son application éventuelle à la recherche du mélange : lait de chèvre et lait de vache. Le Lait, 17, 135. 1937.

[2] R. C. Lopez. Différenciation des laits de chèvre et de vache. Le Lait, 16. 1122.1936 .

[3] G. Hadland et P. Solberg. Serological detection of cows milk added to goat's milk. Meld. Norg. Landbr. Hogsk. Meieri. Adv., 29. 1952, et Dairy Science Abstracts, 15, 74. 1953.

[4] R. Chanda. Carotène in goat liver and colostrum. Biochem. J. 51 (1), IV. 1952 .

[5] D.V. Dearden, K. M. Henry, J. Houston et S. K. Kon. A study of the balance of certain milk nutrients in the making of Cheddar, Cheshire and Stilton cheese, and of their fat during the ripening of the cheese. J. Dairy, Res., 14, 100. 1945.

[6] H. J. Deuel. The lipids. Interscience publishers Ltd. London. 1955. Vol. I, p. 661 .

[7] S. Y. Thомpson et S. K. Kon. - Some application of chromatography in the work of the National Institute for Research in Dairying. VIII e Cong. Intern. Ind. Agric., Bruxelles. 1950. 11, 55.

[8] A. Françors. Recherche sur le comportement biochimique et physiologique de la vitamine A dans la sécrétion lactée. Thèse Fac. Sci., Univ. de Paris. 1948.

[9] P. Lemeland. Recherches sur le dosage des lipoïdes dans le sang et les tissus. Bull. Soc. Chim. Biol., 5, 110, 1923.

[10] P. Karrer et E. Jucker. Carotenoids. Elsevier publishing Company, Inc. New-York, $10 a$, p. 154, 10b, p. 138. 
[11] S. Kuzdzal-Savore. Influence du stade de lactation sur les caractéristiques de la matière grasse du beurre. Ann. l'INRA, Série E, Techno., p. 1, 1956.

[12] Kuzdzal-Savore. Etude de l'influence de la race des vaches laitières sur la composition de la matière grasse du lait et la qualité du beurre. Expérimentation conduite à Echiré. Ann. INRA. Série E. Techno p. 35, 1959.

[13] S. KuzdzaL-Savore. Les limites de variations des indices de la matière grasse du beurre. Ann. Fals. Fraudes, $\mathrm{n}^{0} 575,452.1956$.

[14] A. K. R. MCDowell et F. H. McDowall. The vitamine A potency of New-Zealand butter. J. Dairy Res., 20, 76. 1953.

[15] G. Schwarz, J. Ludwig et E. M. Giessler. Eigenschaften und Zusammensetzung von Kuhmilch in Baden-Wurtemberg. Milchwirts. Forsch. Kiel, 3. 1957.

[16] C. I. Kruisheer et P. C. Den Herder. The vitamin A and caroten contents of dutch butter. Neth. Milk and Dairy J., 6, 109.1952.

\title{
REVUE
}

\section{L'INDUSTRIE LAITIÈRE DANS LE MONDE}

\author{
par \\ G. GENIN \\ Ingénieur E. P. C. \\ ETATS-UNIS
}

\section{Le sérum comme source d'engrais}

A la suite des recherches entreprises à l'University of Wisconsin, il est apparu que le sérum, sous-produit de la fabrication du fromage, pourrait constituer pour certaines cultures de l'Etat de Wisconsin un engrais intéressant.

D'après A. E. Peterson, H. E. Calbert et W. J. Sharratt, qui ont procédé à ces recherches, 3 tonnes de sérum contiendraient autant de substances nutritives pour les plantes qu'une tonne de fumier. Or, dans l'Etat de Wisconsin, on estime qu'il est produit chaque année 2,5 millions de tonnes de sérum dont la moitié est utilisée pour la fabrication d'aliments pour le bétail, mais dont l'autre est perdue en pure perte et pose parfois même des problèmes d'évacuation. L'emploi de ce sérum comme engrais permettrait de simplifier le problème.

\section{Le procédé de Laval pour le traitement du sérum de fromage}

M. W. A. Neumann, Président de la De Laval Separator Co a révélé quelques informations sur un nouveau procédé mis au point par cette firme et qui permet de résoudre un des plus importants 